\title{
Assessment of Wavelet Types in DWT-based Power Quality Disturbance Detection Method
}

\author{
${ }^{1}$ Alper Y1lmaz and ${ }^{* 2}$ Gökay Bayrak \\ ${ }^{1}$ Bursa Technical University, Department of Electrical and Electronics Engineering, Turkey \\ *2 Bursa Technical University, Department of Electrical and Electronics Engineering, Turkey
}

\begin{abstract}
Power quality parameters are the limit values that allow an electrical device to function as intended without significant loss of performance and life expectancy. It is important to detect power quality disturbances quickly and reliably to ensure reliability and continuity of the power system. Today, unlike conventional methods, signal processing-based disturbance and fault detection methods have gained great importance. Wavelet transform (WT)-based disturbance detection methods are the most prominent among these methods. In this study, the performance of the discrete wavelet transforms (DWT)-based disturbance detection method used in the detection of voltage sag and voltage swell events were evaluated for different wavelet types. Process calculation load, response to event signal and performance values were compared for different wavelet types. The results will be an important source for the selection of appropriate wavelets in the methods using WTs.
\end{abstract}

Keywords: Power Quality, Wavelet Transform, Wavelet Functions, Fault Detection, Power Systems

\section{Introduction}

Power quality parameters are the limit values that allow an electrical device to function as intended without significant loss of performance and life expectancy. The main factors affecting power quality are current and voltage harmonics, the frequency drops/rises, voltage sags, voltage swells, voltage interruptions, transient regime events, notches, flickers, and voltage imbalances in the power system [1-3]. Any power system disturbance that causes voltage, current, and frequency deviations can lead to malfunction of the equipment on the consumer side.

Numerous methods based on signal processing have been proposed in the literature for the detection of power quality disturbances. Signal processing-based power quality disturbance detection methods can utilize the time-frequency components of a signal, which is an important advantage over other detection methods. Wavelet transform (WT), Fourier transform (FT), stransform, and Hilbert-Huang transform are basic signal processing-based methods proposed for the detection faults and disturbances [7]. The FT method is missing time information. This method cannot be used to detect all disturbance conditions. Although this shortcoming has been solved by the development of the short-term FT method, it is insufficient because it cannot analyze both low and high-frequency components of the disturbance signal with the fixed scale used in the windowing process [8].

The WT method can investigate discontinuities locally in high-level derivatives and sudden changes in signals where other signal processing methods are insufficient to detect disturbance

*Corresponding author: Address: Department of Electrical and Electronics Engineering Bursa Technical University, Bursa /TURKEY. E-mail address: gokay.bayrak@btu.edu.tr 
and error conditions. Besides, the ability to examine the signal in different frequency bands is an important advantage of this method. Power quality events contain disturbing signals in different frequency bands. Determining the appropriate wavelet type and the number of decompositions to be performed is of great importance in the detection of the disorders. Many studies have discussed the detection of disturbances by the WT-method and used different wavelet types [4$6,12,13]$.

In this study, different wavelet types are evaluated on the performance of the DWT (Discrete wavelet transform)-based event detection method used in the detection of voltage sag and transient events. The DWT-based method is applied to the voltage signals received in real-time via electronic measurement cards with data acquisition cards for different wavelet types. Process calculations load, response to event signal, and performance values were compared when evaluating wavelet types. The results will be an important source for the selection of appropriate wavelets for WT to be used in future developed methods.

\section{Wavelet Transform Theorem and Wavelet Types}

WT, the theorem created by Haar, has been used frequently in fault detection methods and pattern recognition applications in recent years. WT is a signal processing method capable of processing data at different scales and resolutions. In applications, DWT is most commonly used [9]. Using the wavelet function, DWT is calculated from Eq. (2.1).

$$
\operatorname{DWT}(s, b)=2^{-s / 2} \int f(t) \psi\left(2^{-s} t-b\right) d t
$$

Where "s" value corresponds to the scale quantity (frequency), and b value corresponds to the shift (time) value. When performing the DWT conversion, the amount of scale change is of great importance for the accuracy of the analysis. Low-frequency information of the signal is analyzed by approximation coefficients $\left(\mathrm{cA}_{\mathrm{n}}\right)$ while low-frequency information is obtained by detail coefficients $\left(\mathrm{c} D_{n}\right)$ [10]. Mathematical equations of coefficients are given in Eq. (2.2).

$$
c A[n]=\sum_{k} S(k) g[2 n-k] ; \quad c D[n]=\sum_{k} S(k) h[2 n-k]
$$

To be called a wavelet function, the integral of the function must be zero, and the integral of the square of the same function must be one [11].

$$
\begin{aligned}
& \int_{-\infty}^{\infty} \psi(t) d t=0 \\
& \int_{-\infty}^{\infty} \psi^{2}(t) d t=1
\end{aligned}
$$

The waveforms of wavelet functions, which are commonly used in applications, are created using the mathematical model and are shown in Figure 1. 

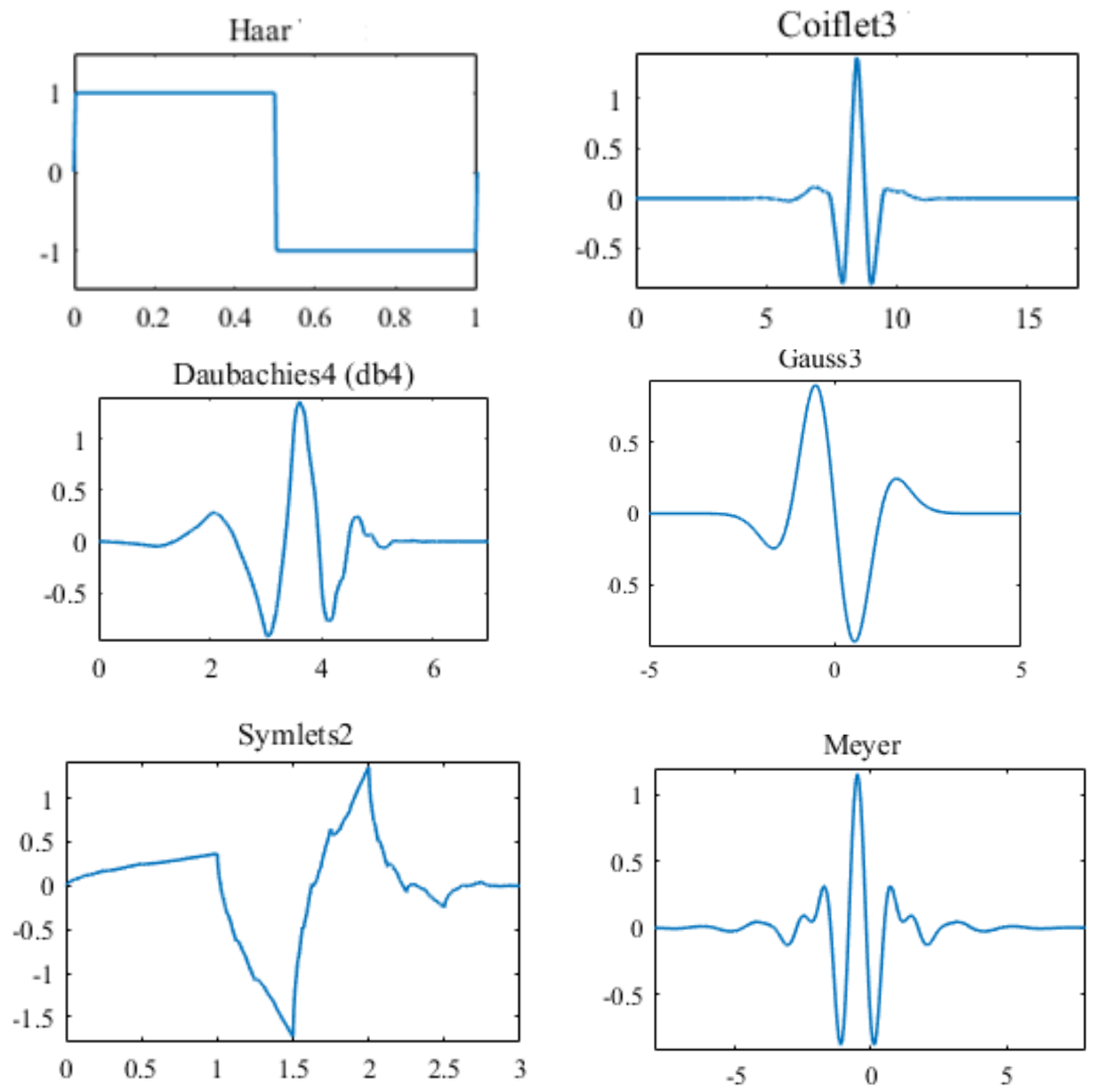

Figure 1. Wavelet functions commonly used in applications

The DWT-based power quality detection process has been started with acquiring the voltage signal at the PCC using the electronic measurement card at $10 \mathrm{kHz}$ sampling frequency. The duration of the examined disturbances signals is 0.2 seconds (2000 points). The 5-level decomposition process performed with different wavelet types and reduced the number of samples at each filter output in half. Different simulation studies have been conducted, and the most appropriate parameters have been determined to define the type of wavelet, wavelet level, sampling frequency, and threshold values to be used in the DWT-based power quality disturbance detection method for fault detection.

In the proposed method, different wavelet functions such as Daubechies (dbn), coiflet (coifn), symlets (symn), Haar and biorthogonal (bi_orn) wavelet types were used. Process calculations load, response to event signal and performance values were compared when evaluating wavelet types. Disturbance signals at both high frequency (transient) and low frequency (voltage sag) were evaluated by performing performance assessment at different decomposition levels. The Labview program in Figure 2 was used to calculate the processing times. 


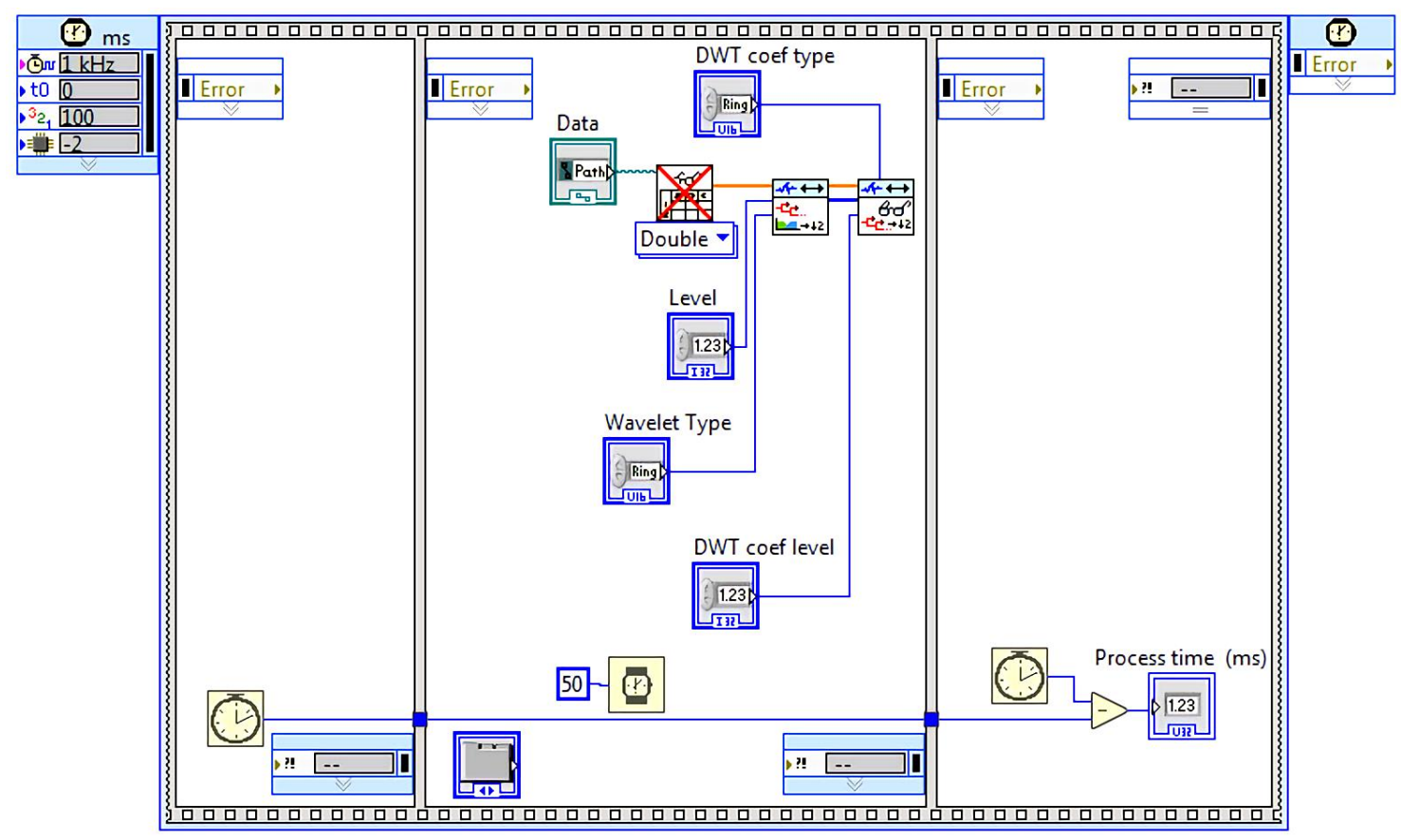

Figure 2. DWT-based method processing time calculation

\section{Results}

In this section, the performance test of different wavelet types is performed for voltage sag and transient events. The detail coefficients are plotted at different decomposition levels. The waveform of the voltage sag is given in Figure 3(a), and the transient event is shown in Figure 3(b). 5-level DWT decomposition is applied to the voltage signals. The responses of the different wavelet functions are given in Figure 4, Figure 5 and Figure 6.

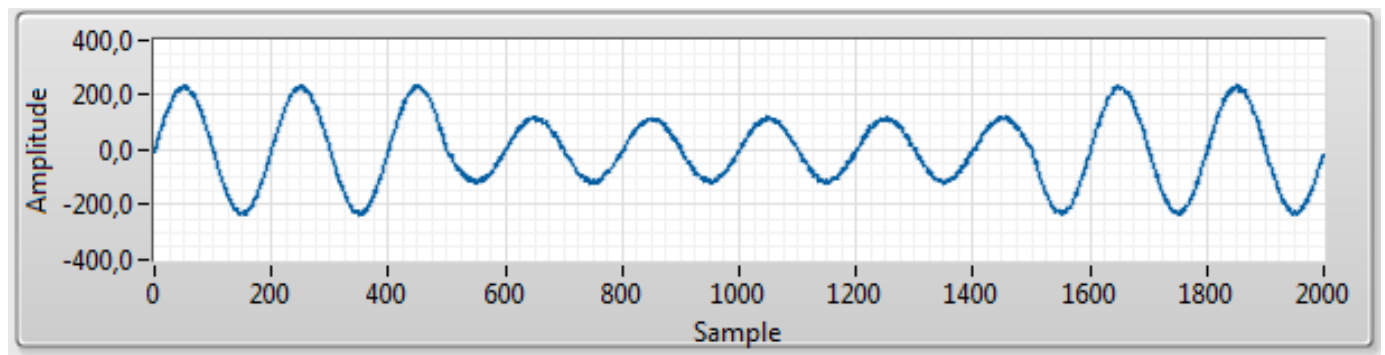

(a)

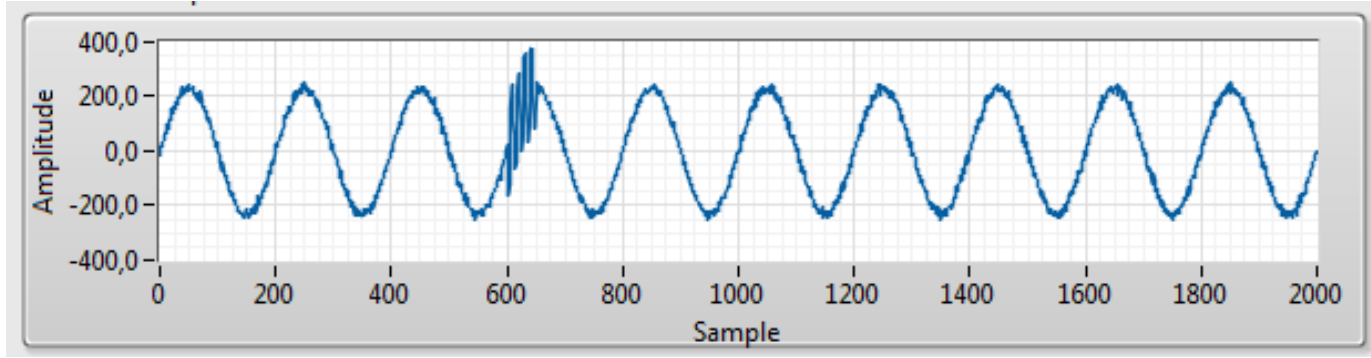

(b)

Figure 3. (a) Voltage sag and (b) transient disturbance events 
DWT Coefficients (db8)

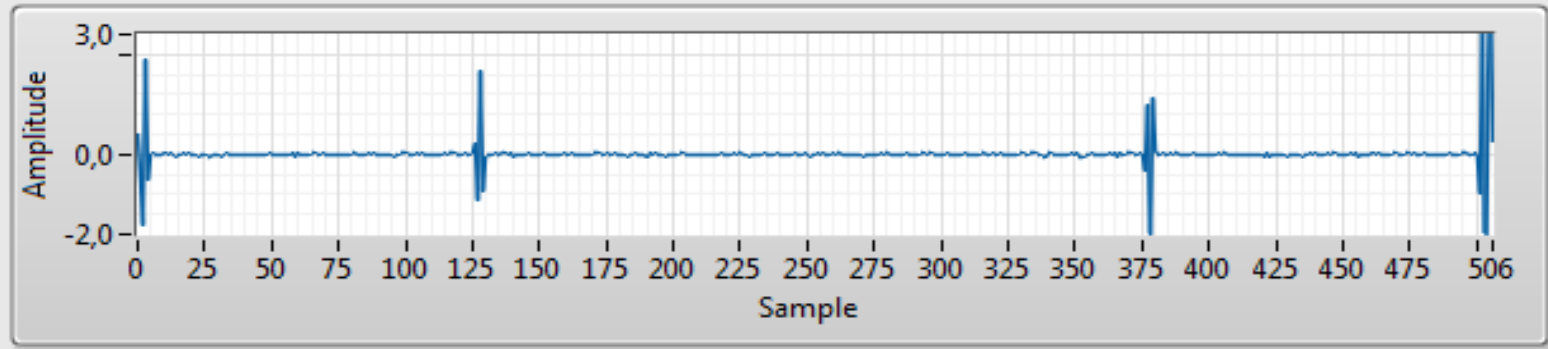

DWT Coefficients (coif4)

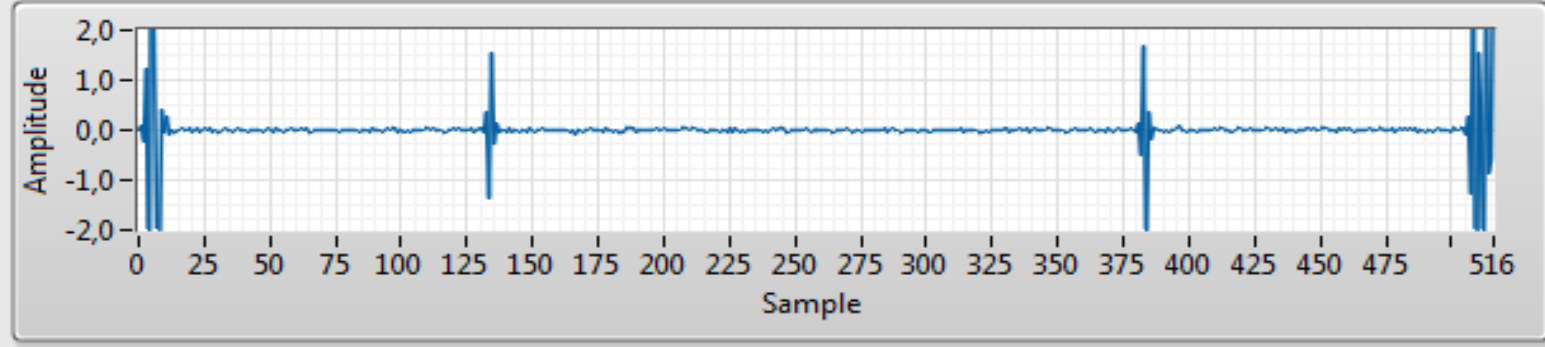

DWT Coefficients (Haar)

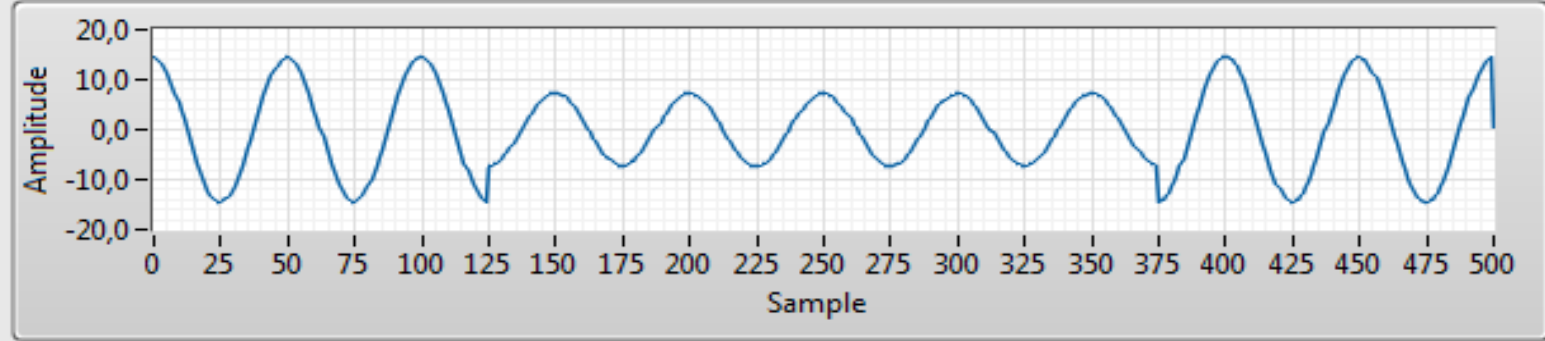

DWT Coefficients (Sym4)

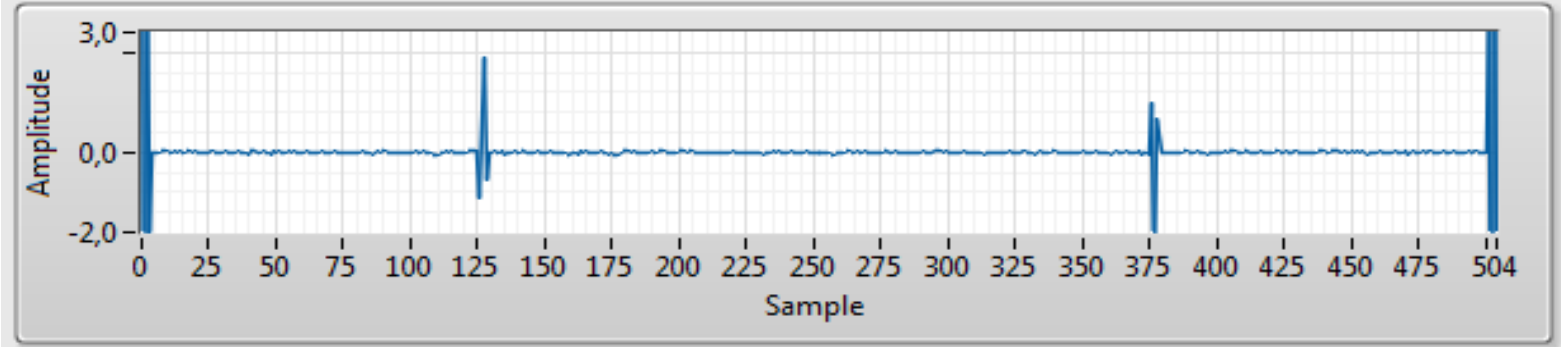

DWT Coefficients (bior1_3)

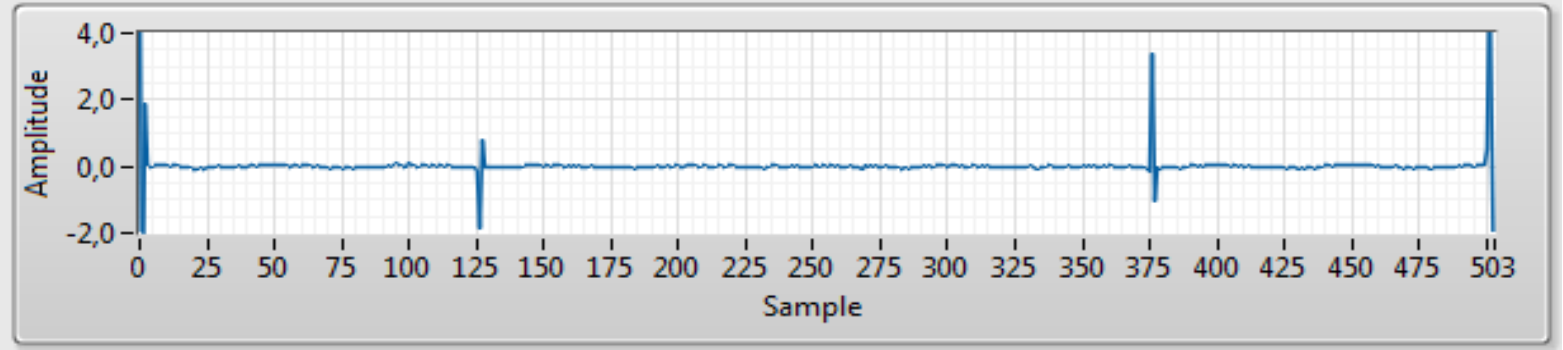

Figure 4. Detail coefficient waveforms as a result of level 2 decomposition (voltage sag) 
DWT Coefficients (db8)

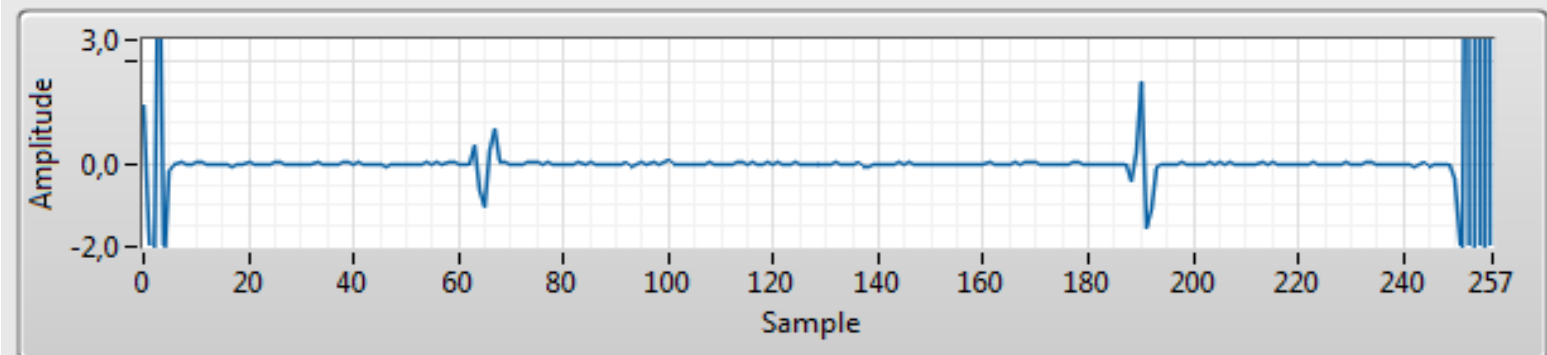

DWT Coefficients (coif4)

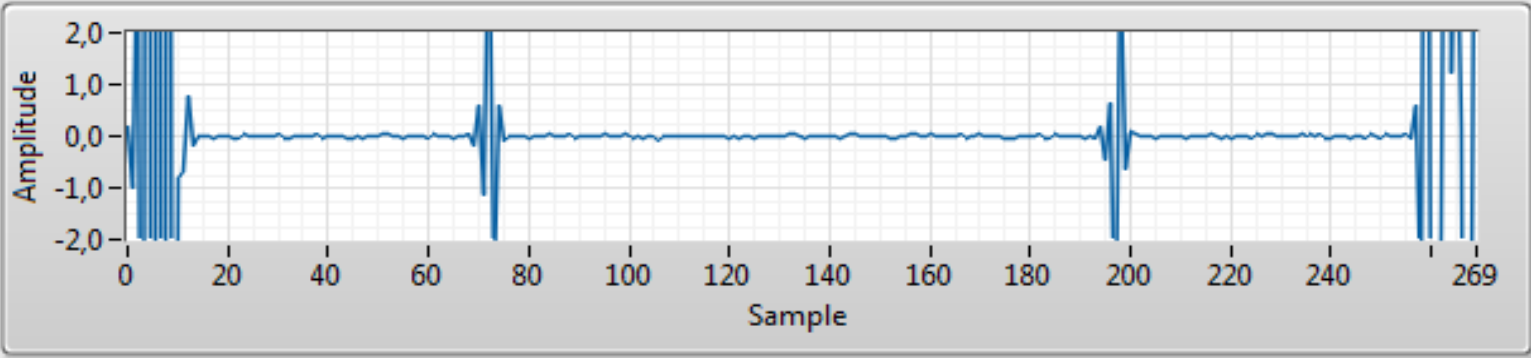

DWT Coefficients (Haar)

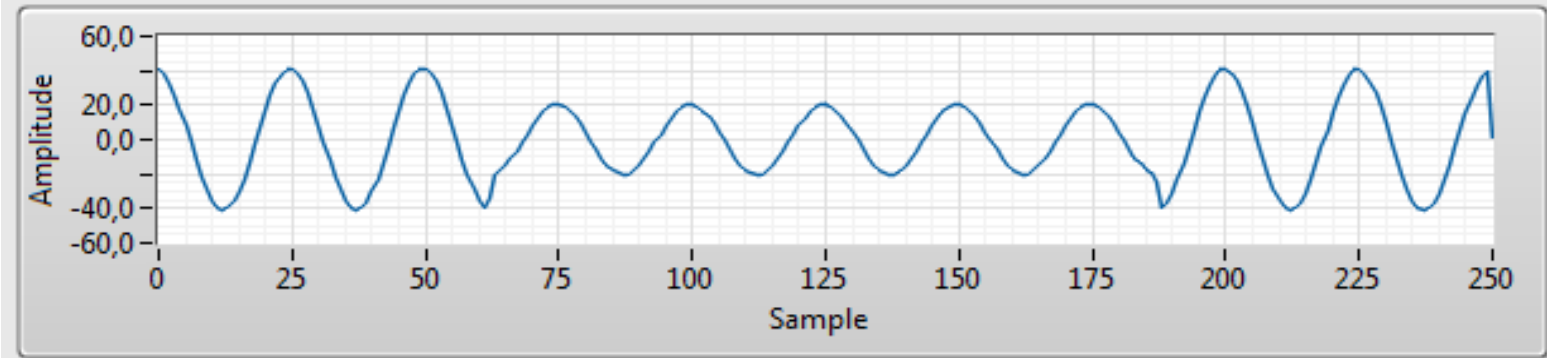

DWT Coefficients (Sym4)

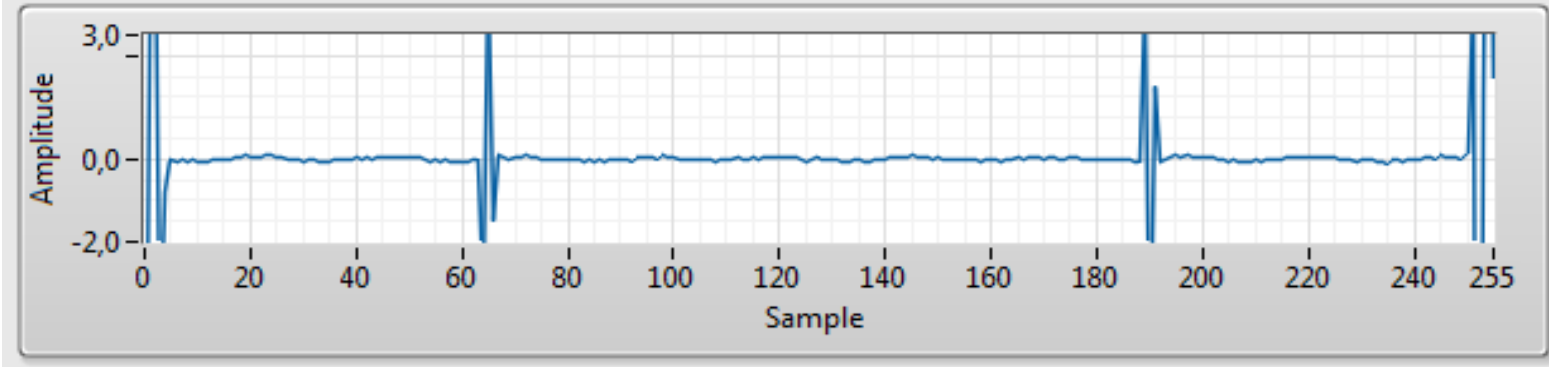

DWT Coefficients (bior1_3)

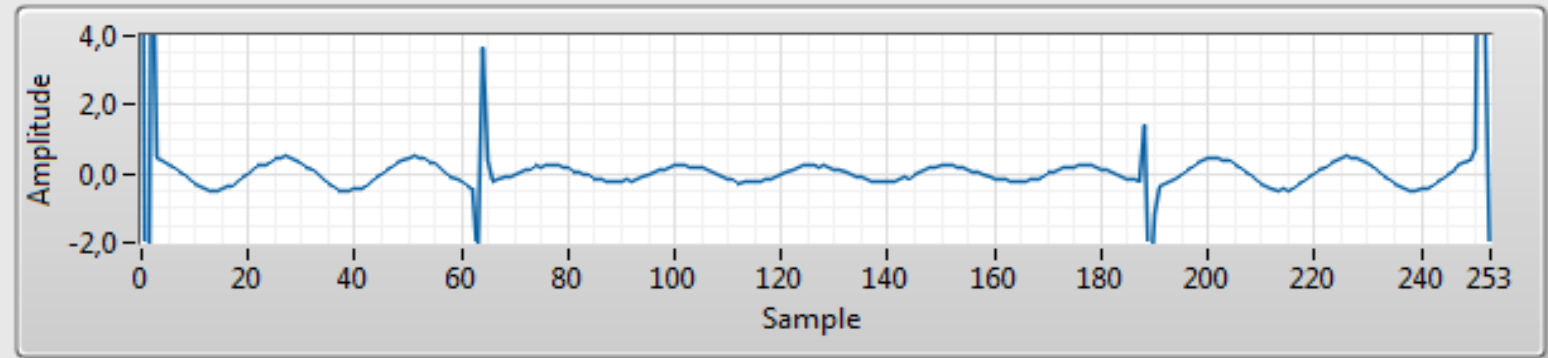

Figure 5. Detail coefficient waveforms as a result of level 3 decomposition (voltage sag) 
DWT Coefficients (db8)

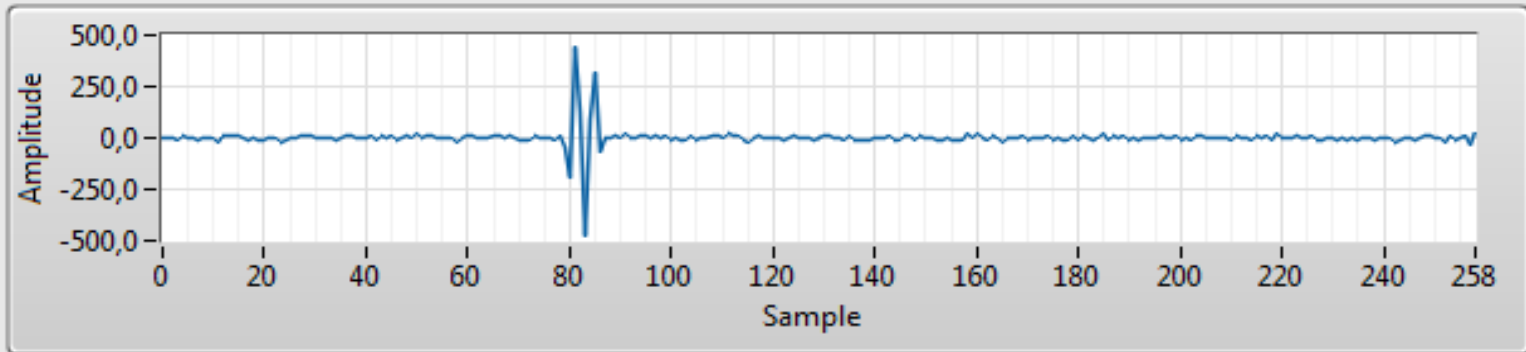

DWT Coefficients (coif4)

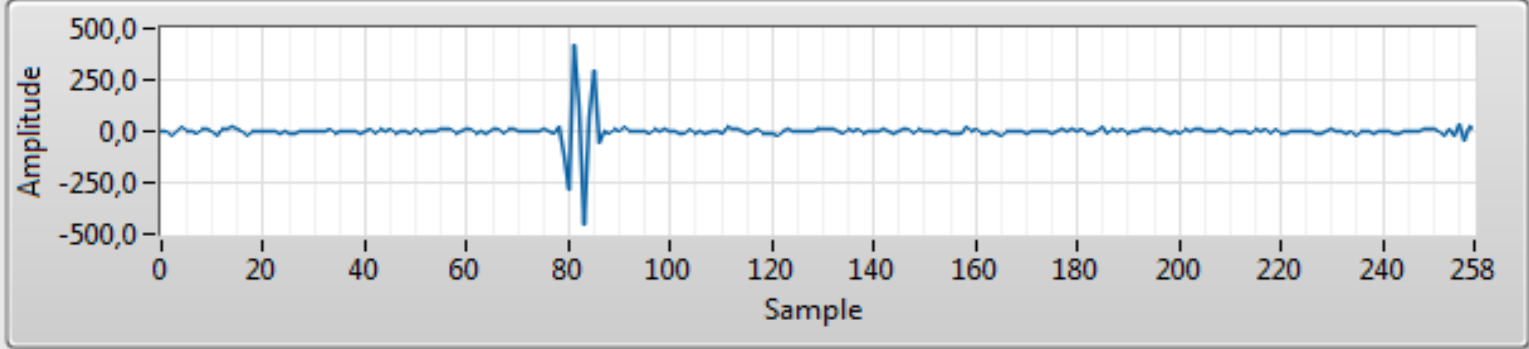

DWT Coefficients (Haar)

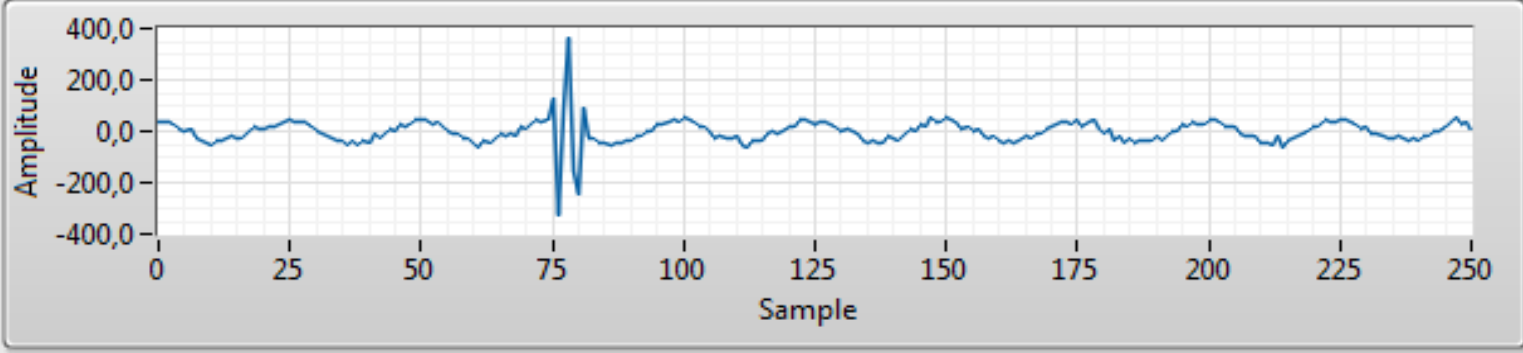

DWT Coefficients (Sym4)

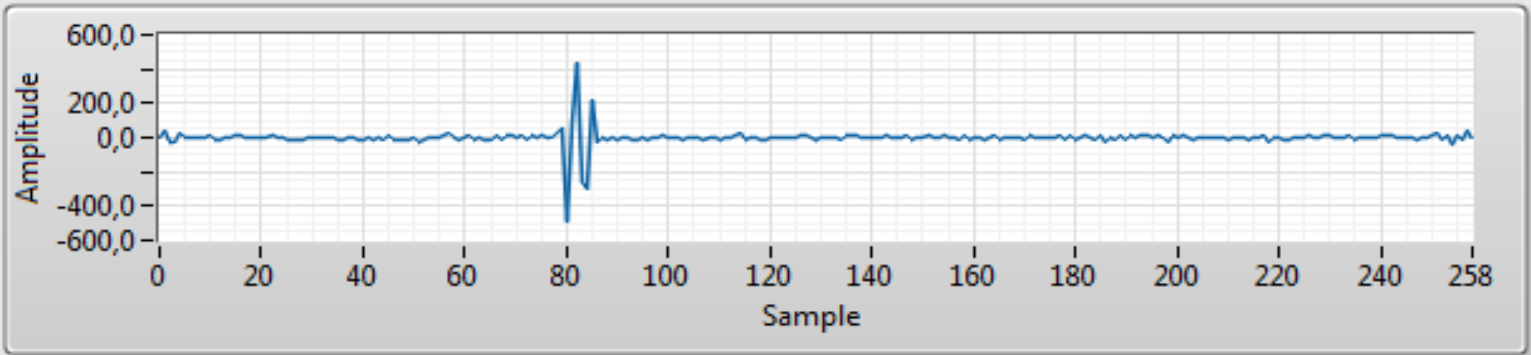

DWT Coefficients (bior1_3)

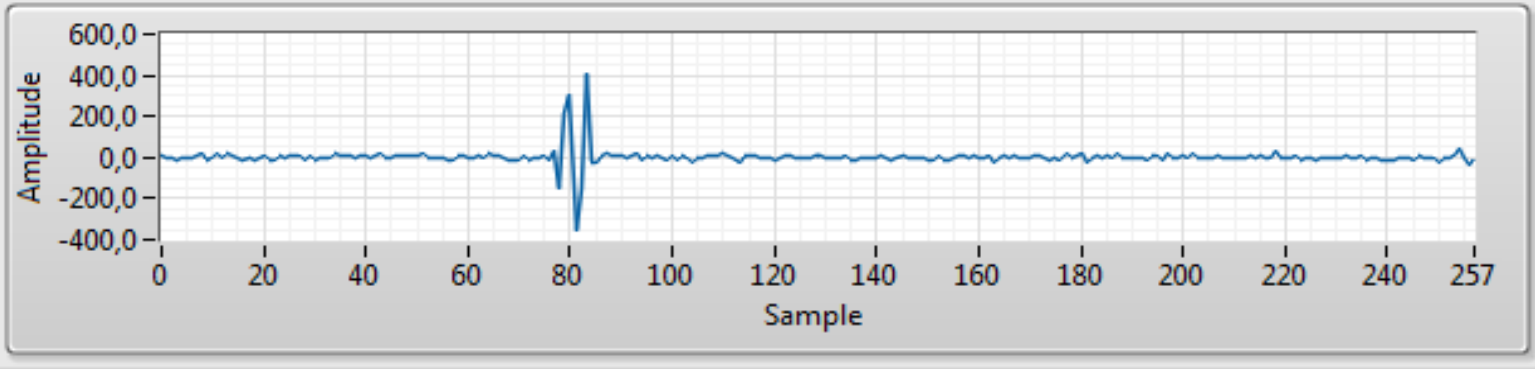

Figure 6. Detail coefficient waveforms as a result of level 3 decomposition (transient event) 
The voltage sag event is given in Figure 3 (a) is started $0.05^{\text {th }}$ ms and lasted 0.15 ms. During this time, the voltage amplitude halved. In Figure 4, the detail coefficients at the second decomposition level at the time of voltage sag are plotted according to different wavelet types. It is possible to determine the fault moment and duration according to the detail coefficients at this level. The detail coefficients at the $3^{\text {rd }}$ separation level in the same fault signal are shown in Figure 5. It is possible to determine the fault moment and duration from the detail coefficients at both levels.

The power quality deterioration in Figure 3 (b) is the oscillating transient event. The event is started at the 0.06th second, and the frequency has increased approximately ten times the nominal. In Figure 6, the detail coefficients are plotted according to the wavelet type at the 3rd level of decomposition during the transient event. Similar to the voltage sag event, it is possible to determine the fault moment and duration properly from the detail coefficients.

\section{Discussion}

In this study, different wavelet types are evaluated on the performance of the DWT-based disturbance detection method, which -applied to the voltage signal at PCC, used in the detection of voltage sag and transient events. The change in wavelet types, process time, and the proper detection of the disturbance moment, although the method is the same, create a difference.

The results of section 3 show that the Haar wavelet function is weak in detecting power quality disturbances. Wavelet types other than the Haar wavelet function can be used to detect the fault moment. Figure 4 shows that the coiflet wavelet function reacts later than Daubechies and symlets wavelet types. The fact that the biorthogonal wavelet function contains a large number of peaks in the graph of detail coefficients in Figure 5 may cause fault detection to be inaccurate. When all graphs are examined, it is seen that the most accurate results are obtained by decomposition with wavelets of Daubechies and symlets. The symlets are nearly symmetrical wavelets proposed by Daubechies as modifications to the db family. The properties of these wavelet families are similar.

\section{Conclusions}

- In this study, the performance of the discrete wavelet transforms (DWT)-based disturbance detection method used in the detection events were evaluated for different wavelet types.

- Process calculation load, response to event signal, and performance values were compared for different wavelet types.

- It is seen that the most accurate results are obtained by decomposition with wavelets of Daubechies and symlets.

- The results will be an important source for the selection of appropriate wavelets in the methods using WTs. 


\section{References}

[1] IEEE Std. 1547. (2015), IEEE standard for interconnecting distributed resources with electric power systems.

[2] IEEE Std, 519. (2014). IEEE recommended practices and requirements for harmonic control in electrical power systems.

[3] IEEE Std, 1159. (2009). IEEE Recommended Practice for Monitoring Electric Power Quality.

[4] Karegar, H. K., \& Sobhani, B. (2012). Wavelet transform method for islanding detection of wind turbines, Renewable Energy, 38(1), 94-106.

[5] Pinto, S. J., \& Panda, G. (2015). Wavelet technique based islanding detection and improved repetitive current control for reliable operation of grid-connected PV systems, International Journal of Electrical Power \& Energy Systems, 67, 39-51.

[6] Naik, C. A., \& Kundu, P. (2013). Power quality index based on discrete wavelet transform, International Journal of electrical power \& energy systems, 53, 994-1002.

[7] Raza, S., Mokhlis, H., Arof, H., Laghari, J. A., \& Wang, L. (2015). Application of signal processing techniques for islanding detection of distributed generation in distribution network: A review. Energy Conversion and Management, 96, 613-624.

[8] Jurado, F., \& Saenz, J. R. (2002). Comparison between discrete STFT and wavelets for the analysis of power quality events. Electric Power Systems Research, 62(3), 183-190.

[9] Strang, G., \& Nguyen, T. (1996). Wavelets and filter banks. Wellesley, SIAM.: WellesleyCambridge Press.

[10] Zhao, W., Song, Y. H., \& Min, Y. (2000). Wavelet analysis based scheme for fault detection and classification in underground power cable systems, Electric Power Systems Research, 53(1), 23-30.

[11] Percival, D. B., \& Walden, A. T. (2006). Wavelet methods for time series analysis (Vol. 4)., New York, Cambridge university press.

[12] Bayrak, G. (2018). Wavelet transform-based fault detection method for hydrogen energybased distributed generators. International Journal of Hydrogen Energy, 43(44), 2029320308.

[13] Yilmaz, A., \& Bayrak, G. (2019). A real-time UWT-based intelligent fault detection method for PV-based microgrids. Electric Power Systems Research, 177, 105984. 